\title{
Building an Intelligent System to Distinguish Russian Printed Letters using
} Artificial Neural Networks

\author{
Jamal S. Majeed \\ College of Computer Science and \\ Mathematics \\ University of Mosul, Iraq
}

Received on: 25/01/2011

\author{
Sura R. Sherif \\ College of Engineering \\ University of Mosul,
}

Iraq

\author{
Osama Y. Mohamed \\ Al-Hadba University \\ College
}

\section{ABSTRACT}

Accepted on: 16/05/2011

In this research, an intelligent computer system is designed for recognizing printed Russian letters by extracting features of the letter by finding the Eigen values which then used for training and testing the artificial neural network used in this work namely, Elman NN. This network is used as a tool for decision making. Data is entered using a flatbed scanner which results in high extensity, fineness and homogeneous BMP extension images. The programs are implemented by Matlab language, the software include image enhancement techniques, image segmentation, resize the segmented image and features extraction dependent on Eigen values .These values are then used to train and test the Elman Neural Network. In this work the pass ratio of recognition up to $90 \%$.

Keywords: Intelligent system, Russian letters, artificial neural network, Elman NN.

$$
\begin{aligned}
& \text { بناء نظام ذكي لتمييز الحروف الروسية المطبوعة باستخدام الثبكات العصبية الاصطناعية }
\end{aligned}
$$

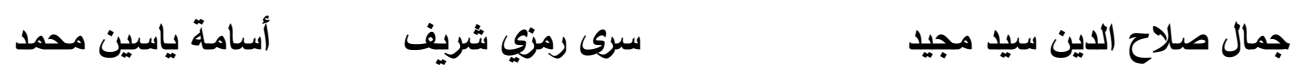

$$
\begin{aligned}
& \text { كلية علوم الحاسوب والرياضيات، جامعة الموصل كلية الهنسة، جامعة الموصل كلية الحدباء الجامعة } \\
& \text { تاريخ قبول البحث: 2011/05/16 } \\
& \text { تاريخ استلام البحث: 2011/01/25 }
\end{aligned}
$$

\section{الملخص}

إن أي نظام لتمييز الأنماط بصورة عامة يجب أن يقوم باستخلاص خصائص مفيدة من النمط المراد

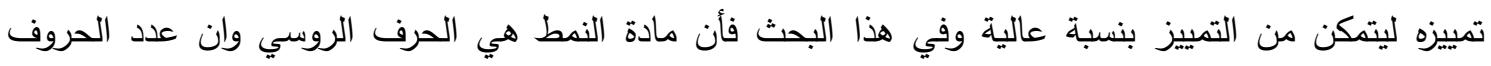

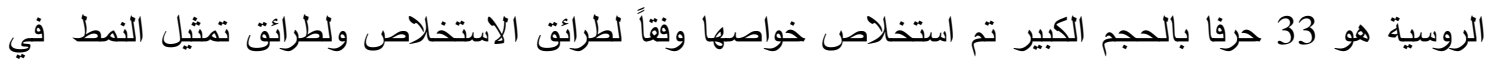

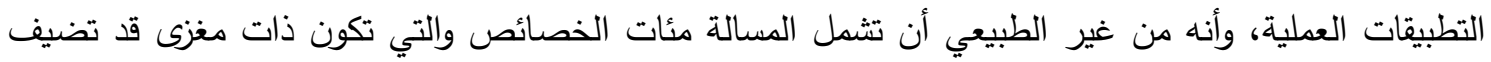

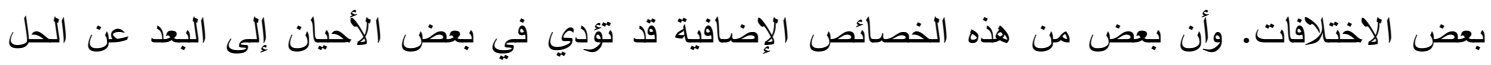

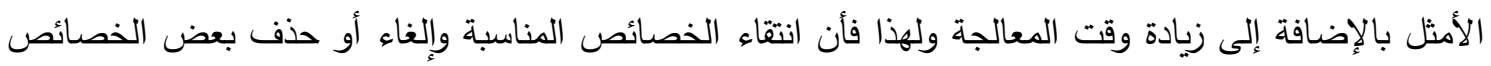


الغير ضرورية ستؤدي إلى تحسين أداء نظام تمييز الأنماط. أن الخصائص المنتقاة في التطبيقات العملية لتمييز

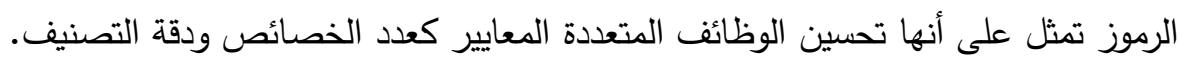

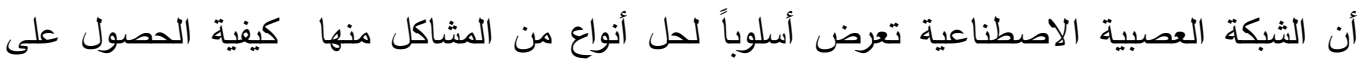
التمييز بصورة أدق خاصة عندما تكون البيانات (الخصائص المستخلصة من الصورة) متقاربة جداً قد تعجز التهن

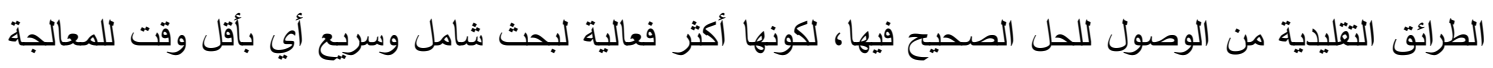
حتى وان كانت المشكلة معقدة. 2- تمييز الرموز البصرية

تمييز الرموز البصرية (Optical Character Recognition (OCR) هي عملية تحويل صورة النص (Text) إلى نص (Text) سواء كان بهيئة مطبوعة أو مكتوب بخط اليد والمؤلف من حروف، أو من أرقام أو من رموز . أن نظام تمييز الرموز البصرية يتألف من ثلاث وحدات أساسية وكما موضح أدناه [6].

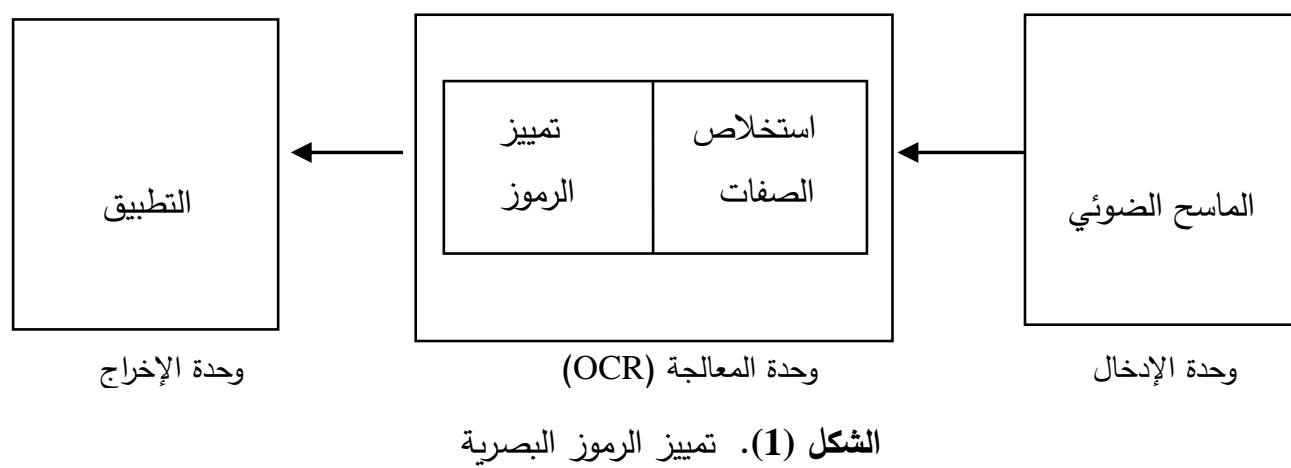

i وحدة الإدخال:- هي عبارة عن تحويل الصورة إلى شكل رقمي قابل للمعالجة من قبل الحاسوب باستخدام أحدى الأجهزة أو المعدات الملحقة بالحاسوب. ويمكن استخدام الماسح الضوئي لفحص وإدخال مختلف أنواع المعلومات المكتوبة والمطبوعة والمصورة والمرسومة والمخطوطة إلى ذاكرة الحاسوب. عن طريق تحويلها إلى بلى إشارات رقمي (Digital Signals) ، قابلة للتخزين في ذاكرة الحاسوب. ii iii. وحدة الإخراج:- عبارة عن نقل نتيجة التمييز إلى التطبيق الذي يستخدمه مثلا إلى قاعدة المعطيات أو برنامج .(Word Processor) 1-2 أن عملية تقطيع صورة الحرف من النص في اللغة الروسية يتم الحصول عليه من صورة النص بتحديد حدود

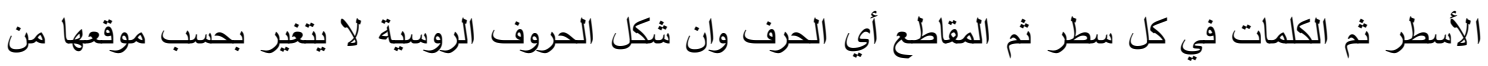

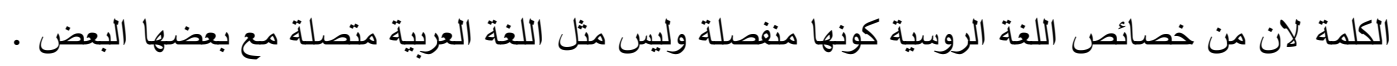
2-2 مراحل تمييز الرموز البصرية أن تمييز الرموز البصرية (Optical Character Recognition (OCR) يتألف من مرحلتين 
المرحلة الأولى:- استخلاص خواص صورة الحرف حيث يتم وصف كل حرف بجملة من الخواص التي تشكل الإدخال لوحدة المعالجة.

المرحلة الثانية:- تمثل التصنيف حيث يتم التعرف على الحرف باستخدام عدة طرق من أهمها:

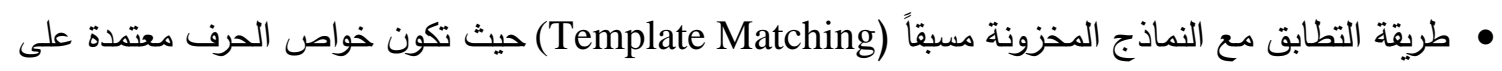
نقاط الصورة الدكونة له (Pixel-Based).

• الطريقة البنيوية حيث يوصف الحرف بجملة من الخواص الهيكلية مثل الخط العمودي، الأفقي، الدائري.

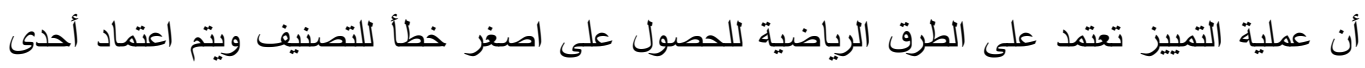

طرائق الذكاء ألاصطناعي في عملية التمييز ومنها الشبكات العصبية الاصطناعية، الخوارزمية الجينية، المنطق

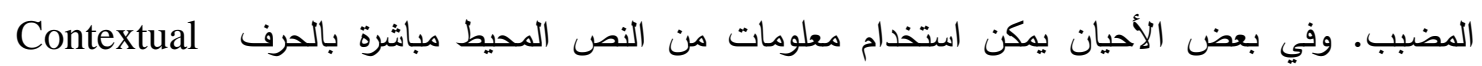
(Processing)

3-2 طريقة التطابق (Template Matching)

تخزن مجموعة من القوالب (متجه الخواص) أو النماذج (Prototype) في الحاسوب حيث يخصص نمط أو قالب واحد لكل حالة مخزونة في الحاسوب. إن عملية التمييز تتم عن طريق إدخال نمط غير معروف

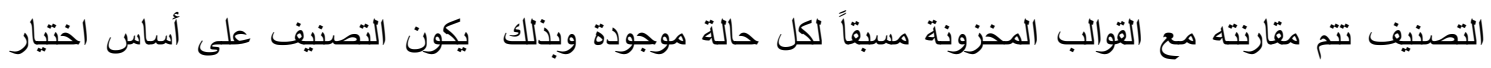

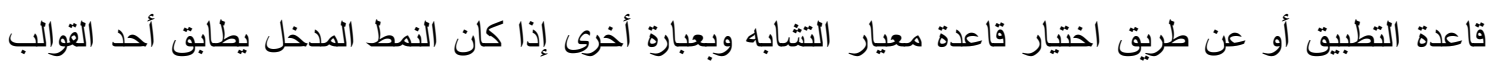
الموجودة أكثر من غيره فان هذا النمط المدخل يصنف عادة على أساس القالب المشابه له لتسهيل عملية المطابقة

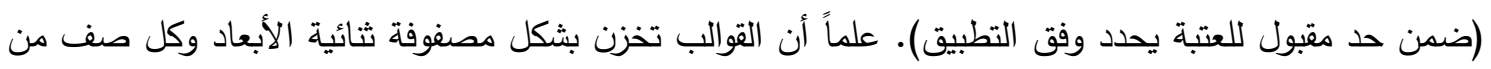

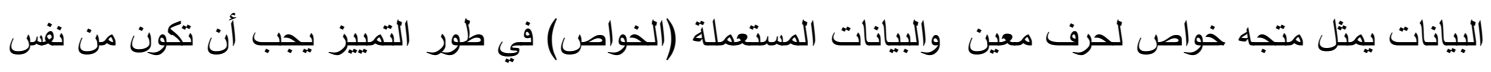

$$
\text { 4-2 أنواع الخواص المستعلة في التدريب [6]. }
$$

أن تمييز الرموز البصرية (Optical Character Recognition (OCR) يعتبر من أوسع التطبيقات

لتمييز الأنماط البصرية كونه يمثل جزء من هذه الأنواع في تعامله مع العمليات البصرية للرمز لتمييز صورة

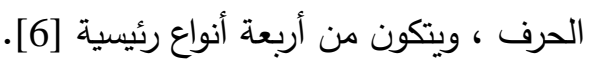

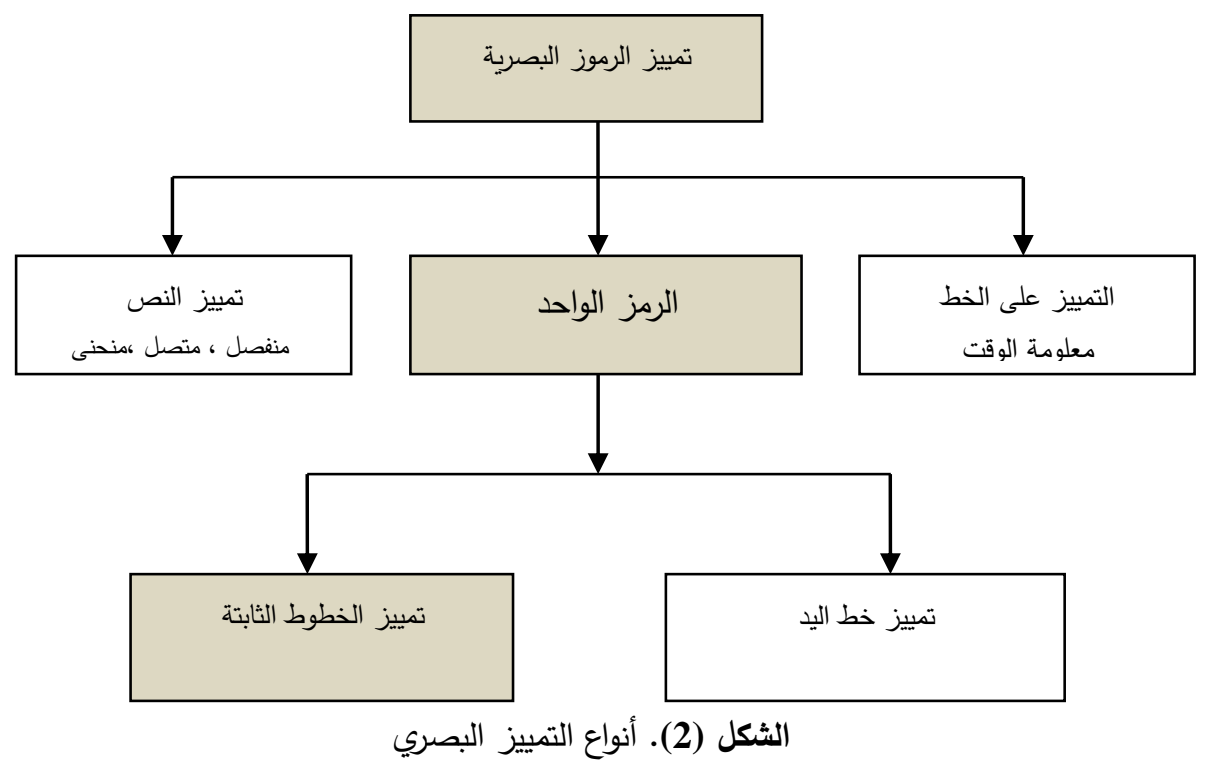


في الثكل (2) الصناديق المضللة تم اختيارهـا كأنواع مستعملة في هذا البحث لأنها حروف روسية

مطبوعة وليست بخط اليد.

• تمييز الرموز الثابتة (Fixed Font Recognition):- هو تمييز خط معين محدد وحيث يكون التمييز محدد بهذا الخط فقط (OCR-A,OCR-B).

• التمييز عند الزمن الحقيقي (On -Line recognition):- هو التمييز ألآني لكل رمز وتحويله من هيئة صورية إلى هيئة نصية باستخدام برمجيات تمييز الرموز الضوئية (OCR) المتعلقة بالتطبيق.

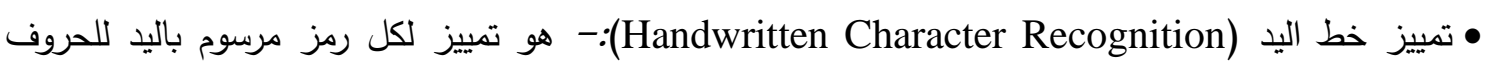

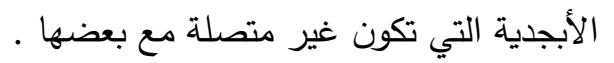

• التمييز النصي (Script Recognition):- هو التمييز للكتابة بخط اليد بدون أعاقة والتي من المكن أن تكون متصلة أو منحنية.

5-2

أن أنظمة تمييز الرموز البصرية في التطبيقات يتم تقسيمها إلى قسمين أنظمة تمييز خاصة وأنظمة تمييز عامة

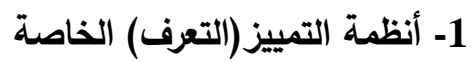
أن أنظمة التمييز أو التعرف الخاصة تقوم بالتعرف على النصوص المحددة لاستخدامها في تطبيق معين

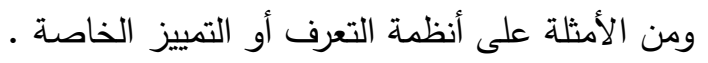
• قراءة العناوين لتصنيف الرسائل حيث تتم قراءة الرمز البريدي وتصنيفه. • قراءة الثيكات للتعرف على قيمة ورقم الحساب. • قراءة بطاقات الطائرات حيث يتم التعرف على رقم البطاقة ويقارن مع قاعدة البيانات الخاصة بالحجز •

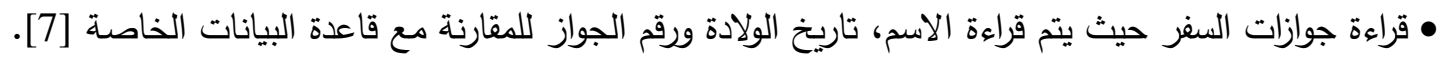

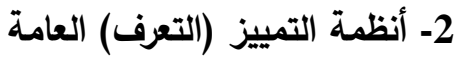
يعتبر هذا النوع من الأنظمة أكثر شمولية وغالباً تستخدم في التطبيقات المكتبية وفي نظام قراءة آلية للمكفوفين بعد ربطها مع نظام لتركيب الكلام [4]. 6-2 - 6 - 6 - 6 - 6روف الروسية أن الحروف الروسية يبلغ عددها 33 حرفا وتمثل بحالتين هما حالة الحروف الكبيرة وحالة الحروف الصغيرة وهي تثابه بذلك الحروف الانكليزية وكما مبين في الجدول (1). الجدول (1). الحروف الروسية

\begin{tabular}{|c|c|}
\hline ЁЙЦУКЕНГШЩЗХЬФЫВАПРОЛДЖЭЯЧСМИТЬБЮ & الحروف الكبيرة \\
\hline ёйцукенгшщзхъфывапролдэ & غيرة \\
\hline
\end{tabular}


1 شبكة أيلمان العصبية الاصطناعية Elman Neural Network

الثبكات العصبية هي تقنيات حسابية مصممة لمحاكاة الطريقة التي يؤدي بها الدماغ البشري مهمة معينة، وذلك عن طريق معالجة ضخمة موزعة على التوازي، ومكونة من وحدات معالجة بسيطة، هذه الوحدات ما

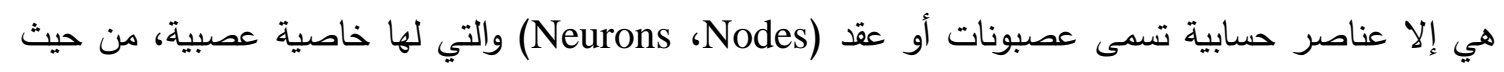
أنها تقوم بتخزين المعرفة العملية والمعلومات التجريبية لتجعلها متاحة للمستخدم وذلك عن طريق ضبط التهات الأوزان. ويتم ربط هذه العصبونات بواسطة بعض أنواع الربط والتي تحمل بيانات رقمية، ويتم تشفيرها بطرائق متتوعة.

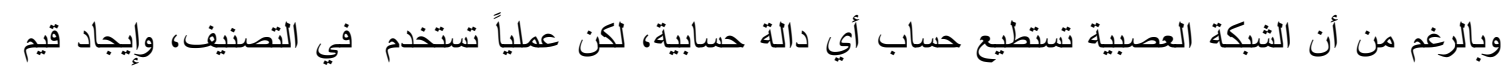

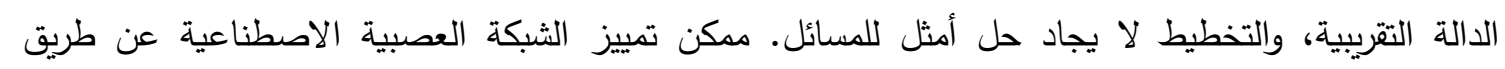
استخدام العديد من الأسماء مثل الإتصالاتية، نظام الذكاء العصبي والمعالجة المتوازية الموزعة [15]. أن شبكة أيلمان العصبية الاصطناعية (Elman) تتكون من طبقتين ويتم اعتماد تغذية الانتشار الخلفي

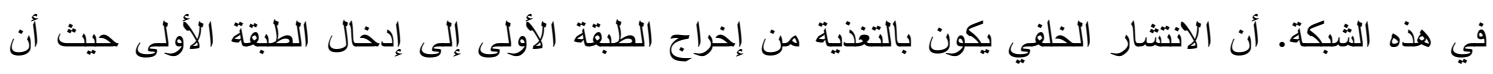

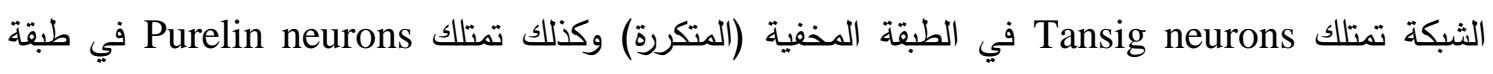
الإخراج. أن الطبقة المخفية يجب أن يكون لديها خلايا عصبية بما فيه الكفاية وأن هذه الشبكة تختلف عن الثبكات التقليدية التي تمتلك طبقتان حيث تمتاز بوصولها إلى الحل الأمثل لأنها تعيد إخراج الطبقة المخفية إلى لى لئه

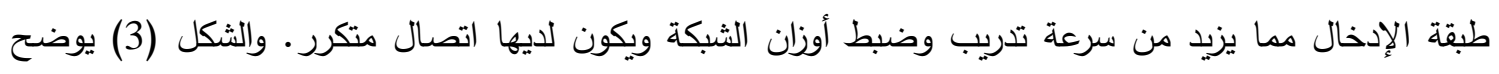
شبكة Elman

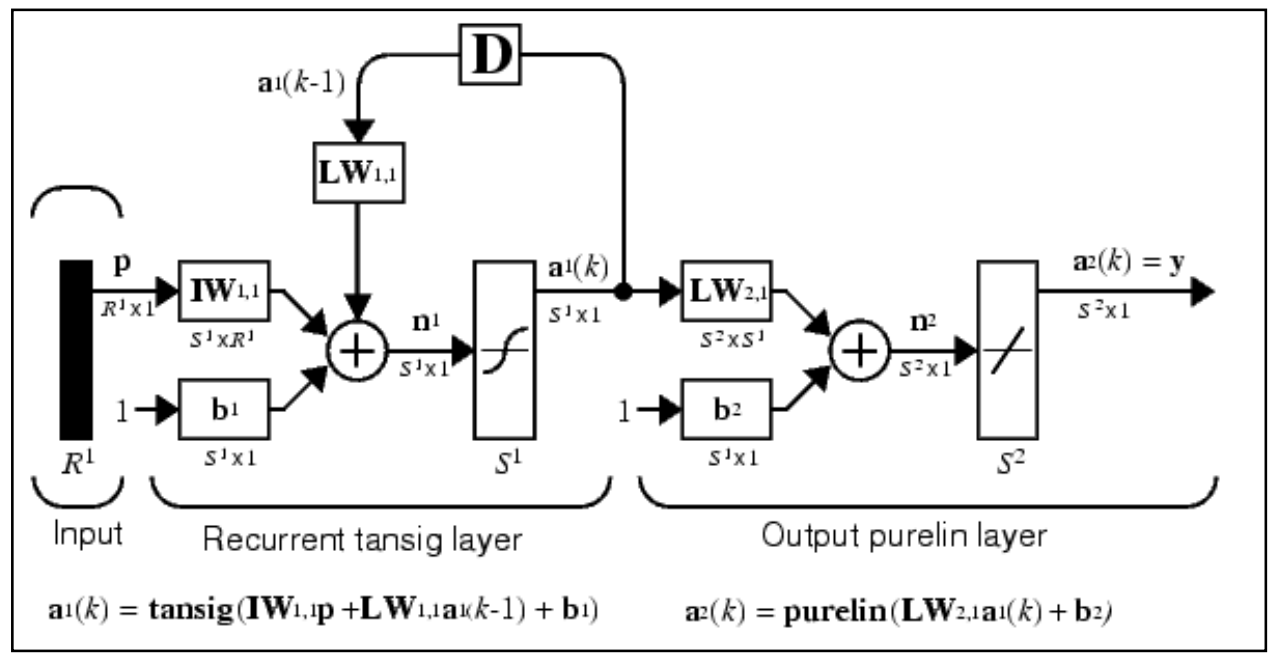

الثكل (3). معمارية شبكة Elman

\section{Elman 1-3 التدريب لشبكة}

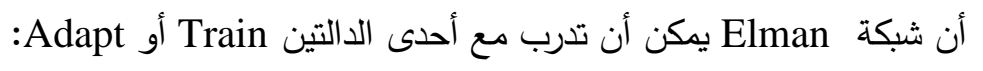

عند استعمال الدالة Train لتدريب شبكة

1. سلسلة الإدخال الكاملة تقدم إلى الثبكة ونواتجها تحسب وتقارن بسلسلة الهدف لتوليد سلسلة خطاً.

2. الخطاً هو Back propagated وذلك لإيجاد ميول الأخطاء لكل وزن وانحياز حيث هذه الميول في الحقيقة

$$
\text { هي تقريبية. }
$$


3. ثم يستعمل هذا الميل التقريبي لتعديل الأوزان بدالة التتريب Back propagated المختارة والدالة يفضل أن

تكون Traingdx وعندما يتم استعمال الدالة adapt لتدريب الثبكة Elman فسيحدث ما يلي :

1. سلسلة الإدخال الكاملة تقدم إلى الثبكة ونواتجها تحسب وتقارن بسلسلة الهدف لتوليد سلسلة الخطاً. 2. الخطاً هو Backpropagated وذلك لا يجاد ميول الأخطاء لكل وزن وانحياز حيث هذه الكيول في الحقيقة

$$
\text { هي تقريبية . }
$$

3. ثم يستعمل هذا الميل التقريبي لتعديل الأوزان بدالة التتريب Back propagated المختارة والدالة يفضل أن

تكون learngdm.

Train

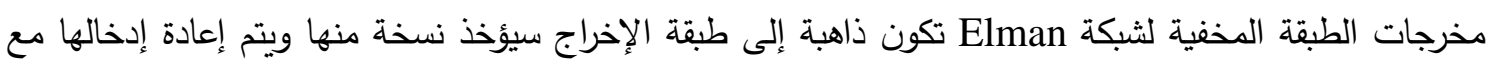

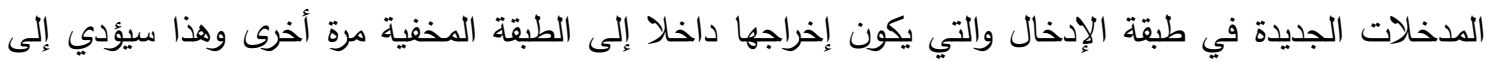

سرعة وصول الثبكة إلى الاستقرارية كما في الثكل (4).

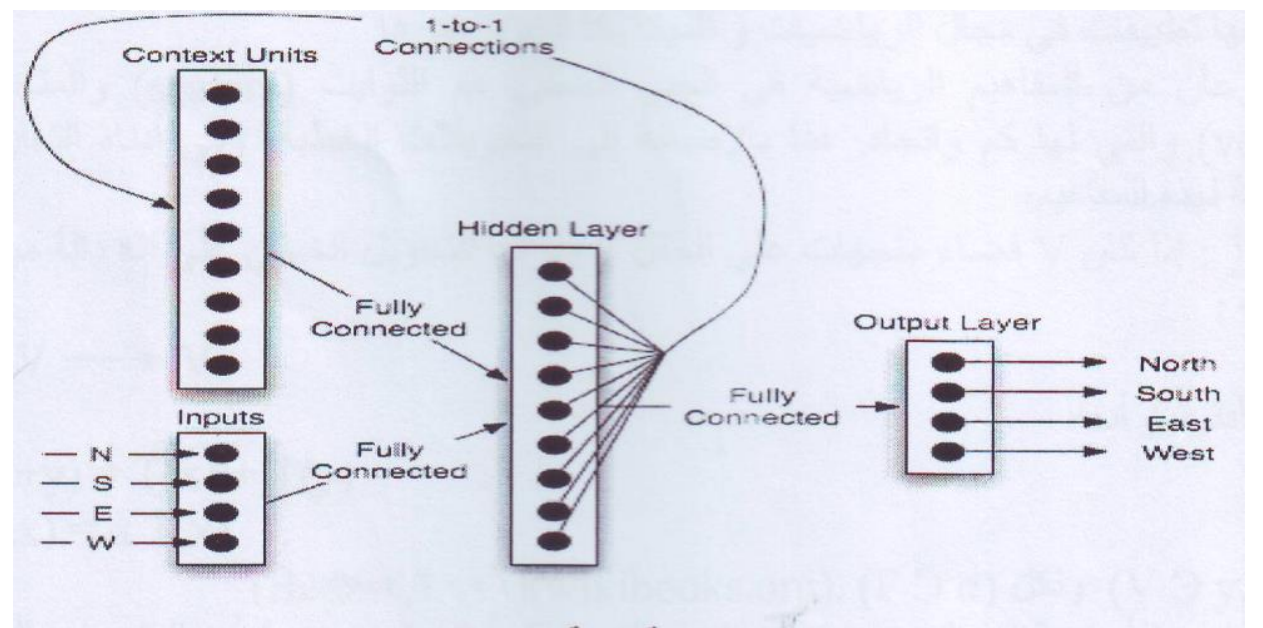

الثكل (4). معمارية شبكة Elman

4- القيم الذاتية والمتجهات الذاتية والمعادلة المميزة

أن علم الرياضيات وضمن مادة الجبر الخطي تدرس بعض الدفاهيم الرياضية مثل التحويلات الخطية

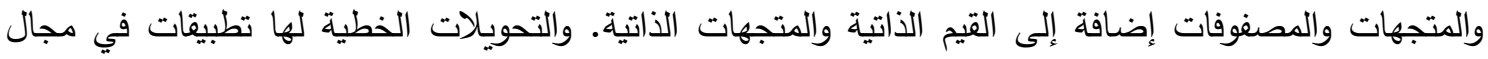

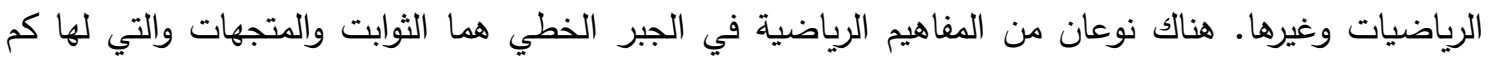
واتجاه. هذا بالإضافة إلى التحويلات الخطية وفي أدناه التعاريف الرياضية لهذه الهفاهيم [9]. تعريف 1: إذا كان V فضاء متجهات على الحقل F يعرف التحويل الخطي على أنه دالة من V إلى نفسه : $T: V \rightarrow V$

$\mathrm{T}(\mathrm{X}+\mathrm{Y})=\mathrm{T}(\mathrm{X})+\mathrm{T}(\mathrm{Y})$

$\mathrm{T}(\alpha \mathrm{X})=\alpha \mathrm{T}(\mathrm{X})$ يحقق الفقرتين أدناه

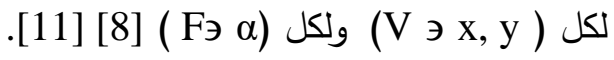


ومن المفاهيم الأساسية في الموضوع هو مفهوم القيم الذاتية Eigen Value والمتجهات الذاتية Eigen Vectors

والتي تعرف بالثكل (5).

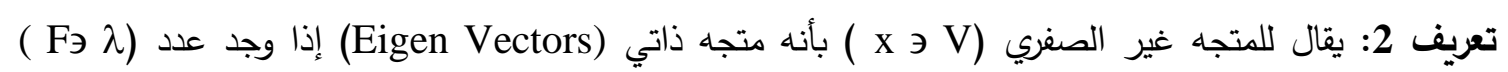

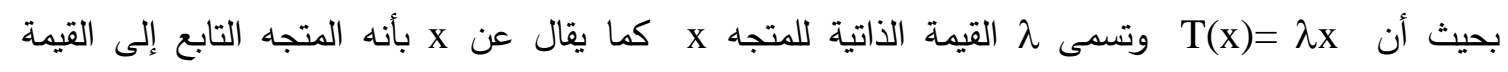

أن الوصف الهندسي لمعادلة القيمة الذاتية (T(x)= Ax) يعني بالضبط أن المتجه الذاتي عندما يقع تحت تأثير التحويل T فأنه يتغير كماً وقد يحافظ على اتجاهه (عندما يكون $\lambda$ > 0) أو ينعكس اتجاهه بالاتجاه المعاكس والثكل (5) يوضح تغير في مقدار المتجه دون تغير في اتجاهـ.

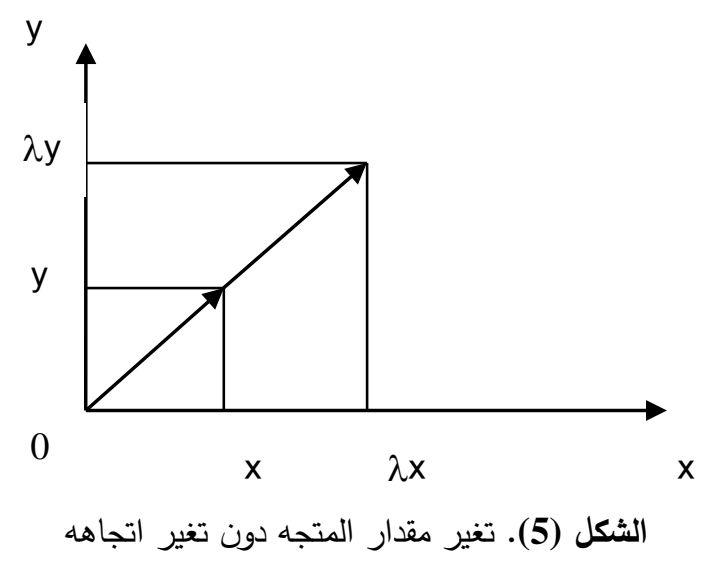

وبذلك فأن القيمة الذاتية تمثل كمية الامتداد أو الانكماش الذي يطرأ على المتجه x عندما يتحول بتأثير T فإذا

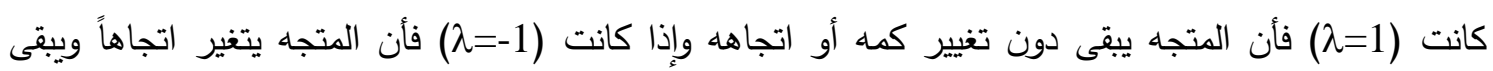

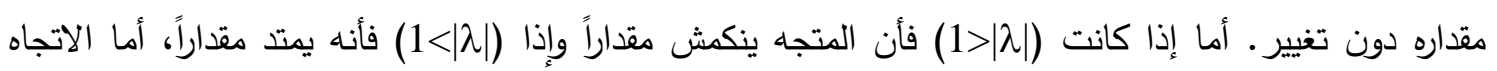

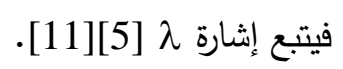

تعريف 3: يقال للتحويل المعرف بالثكل (Ix=x) لكل (Vэ x) بأنه تحويل الوحدة [9] [11]. تعريف 4: يقال للفضاء الذي يتكون من عدد من المتجهات الذاتية التابعة لنفس القيمة الذاتية بأنه فضاء ذاتي ,أي

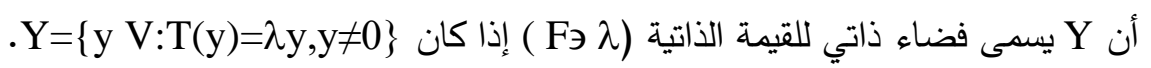
ومن الجدير بالذكر بأن المتجهات في حقل الرياضيات يمكن أن تمثل عدد من المفاهيم الرياضية مثل الأزواج

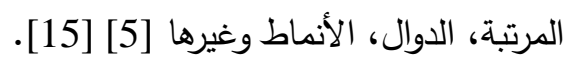

1-4

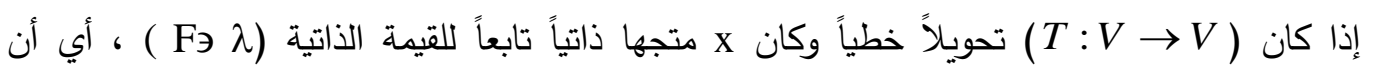

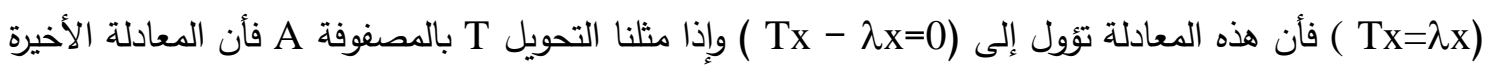
تمثل بالمعادلة

$A x-\lambda x=0$

أو 


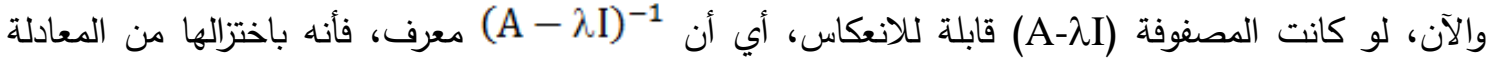

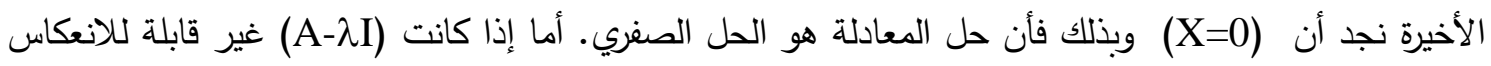
فأن الاخيرة نجان

تعريف 5: إذا كان ( $)$ هي المصفوفة التي تمثل التحويل T M فأن المعادلة

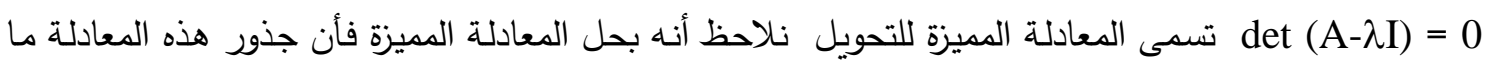
هي إلا القيم الذاتية للتحويل T وبالتالي نجد المتجهات الذاتية التابعة لهذه القيم [11][11].

5- تمييز الأنماط في الثبكات العصبية الاصطناعية Patterns Recognition in Artificial Network أن الثبكات العصبية الاصطناعية تستخدم في تمييز الأنماط. وتمييز الأنماط هي العملية التي تتم على البيانات للحصول على معلومات مرتبة لتصنيف هذه البيانات.

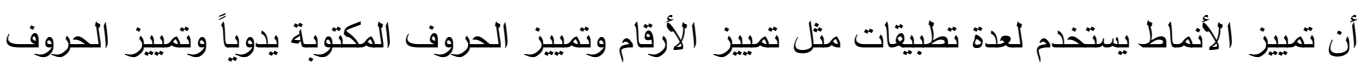

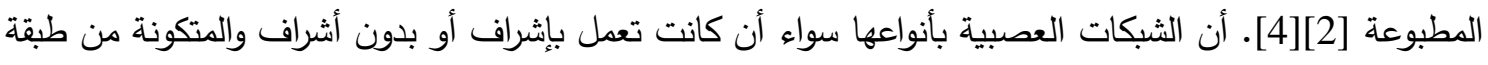
واحدة والمتكونة من عدة طبقات استطاعت وبنجاح تطبيق مختلف التصانيف ومسائل التمييز ـ فليس من الضرورة

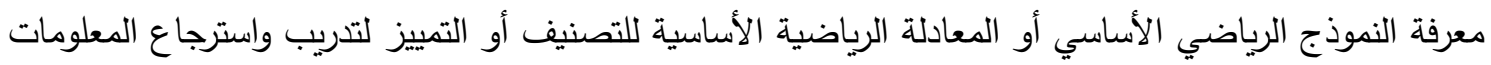

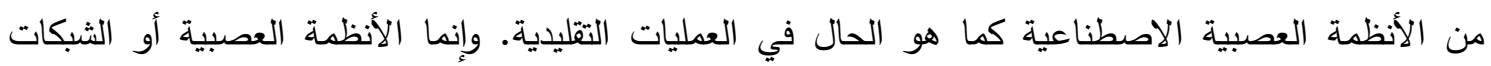
العصبية تتطلب معرفة زوج التدريب أي الإدخالات والإخراج المطلوب ومعرفة معمارية الثبكة وخوارزمية الثبكة العصبية التي تستطيع أن تتكيف مع معاملات الثبكة أي (Parameters) للحصول على حل مقبول والحل

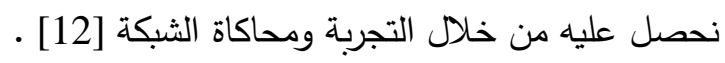
6- استخلاص الصفات إن استخلاص الصفات من المراحل المهمة في نظام تمييز الأنماط حيث تأخذ الصفات التي تحقق دقة عالية في التمييز وسرعة في المعالجة، وأن استخلاص الصفات يجب أن تحقق أهداف صحيحة وتشمل أولاً: اختيار الصفات المستخلصة من النمط يجب أن تكون فعالة في مرحلة التصنيف.

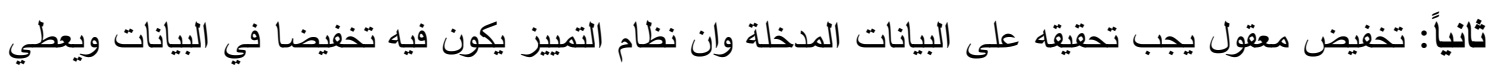

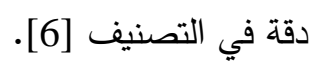
7- نظام تمييز الحروف الروسية باستخدام الثبكات العصبية في هذه الفقرة سيتم شرح المراحل لتنفيذ نظام التمييز الصوري للحروف الروسية والثبكة العصبية الاصطناعية المستخدمة كأداة لتمييز وكيفية تحويل صورة الوثيقة إلى وثيقة نصية نستطيع التعامل معها والتغيير والتعديل عليها والصورة المستخدمة في هذا البحث وهي (Bit map image) من نوع 8 بت ومن ثم الانتقال إلى مراحل نظام تمييز الحروف والثبكة العصبية وثم مرحلة تتفيذ البرنامج والثكل (6) يوضح مثال لصورة الأحرف

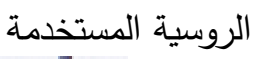
Ёйцукенгшщзхьфывапролданяячсмитьбюю الثكل (6). مثال لصورة الأحرف الروسية المستخدمة 
في هذه المرحلة يتم إدخال الصورة ذات الامتداد (Bmp.) من اي جهاز ماسح ضوئي وضمن كثافة

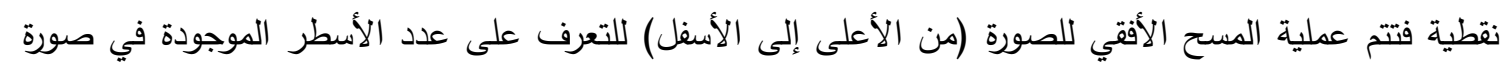

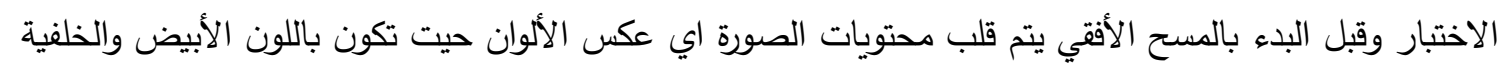

باللون الأسود.

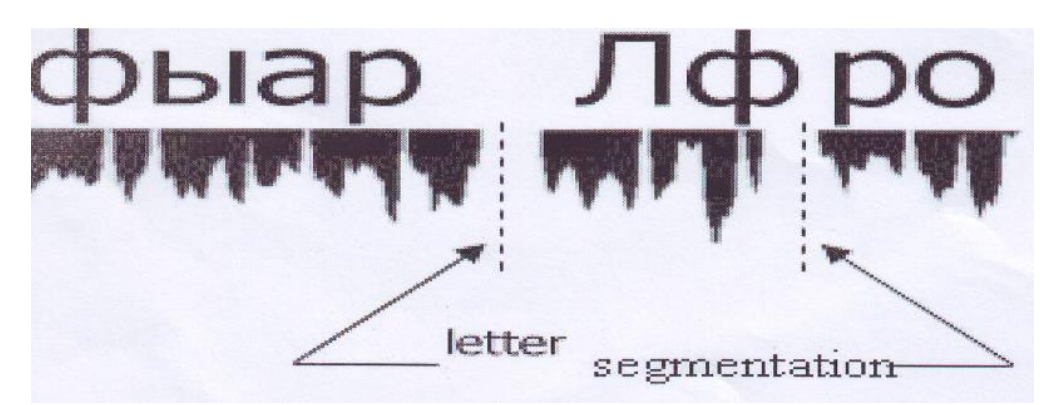

الثكل (7). شكل الحرف الروسي بعد عملية المسح الأفقي

2-7

بعد عملية المسح الأفقي تأتي عملية المسح العمودي والتي تعني تقسيم الأسطر إلى حروف ليتسنى استخلاص خواص كل حرف على حدا وهي تثابه عملية المسح الأفقي.

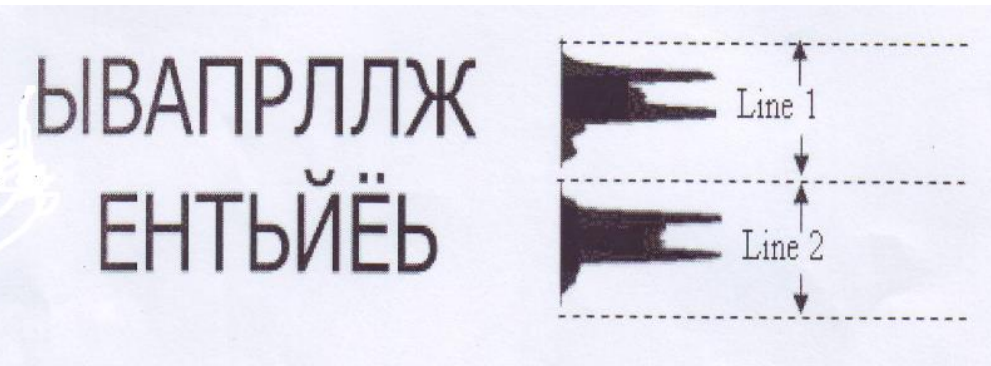

الثكل (8). شكل الحرف الروسي بعد عملية المسح العمودي

3-7 حساب القيم الأتية لصورة الأحرف الروسية

بعد اقتطاع صورة الحرف الروسي بالاعتماد على المسح الأفقي والمسح العمودي نقوم بتغير صورة الحرف لتكون ضمن مصفوفة مربعة الأبعاد لحساب القيم الذاتية لكل حرف حيث لا يمكن حساب القيم الذاتية لمصفوفة التئي مستطيلة. وبما أنه القيم الذاتية تحتوي على أعداد حقيقية وأعداد مركبة فعمدنا إلى تحرير العدد المركب من قيمة لإدة

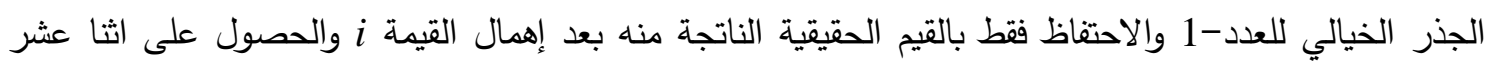
قيمة تكون هي الإدخالات للشبكة العصبية أيلمان لغرض التمييز . الثكل (10) يوضح ذلك كما أن القيم الذاتية

لجميع الأحرف الروسية موجودة في الملحق (1). 


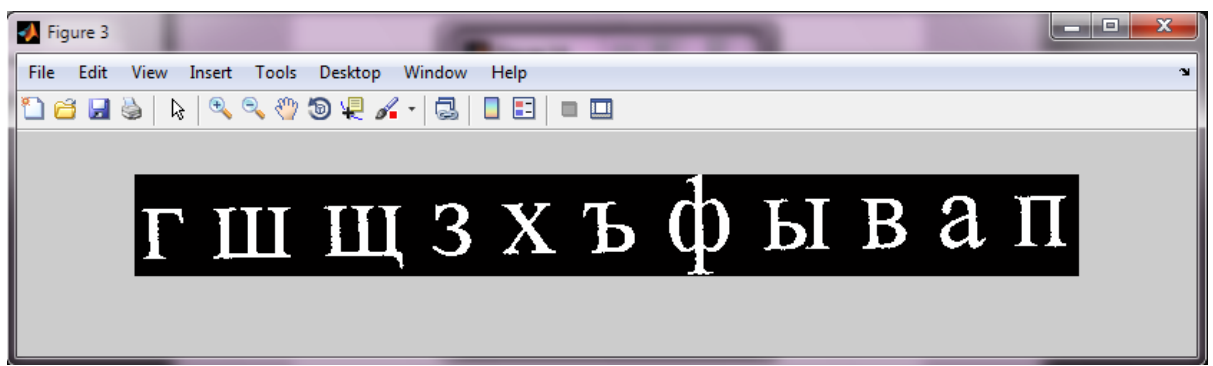

$$
\text { الثكل (9). صورة الحرف الروسي }
$$
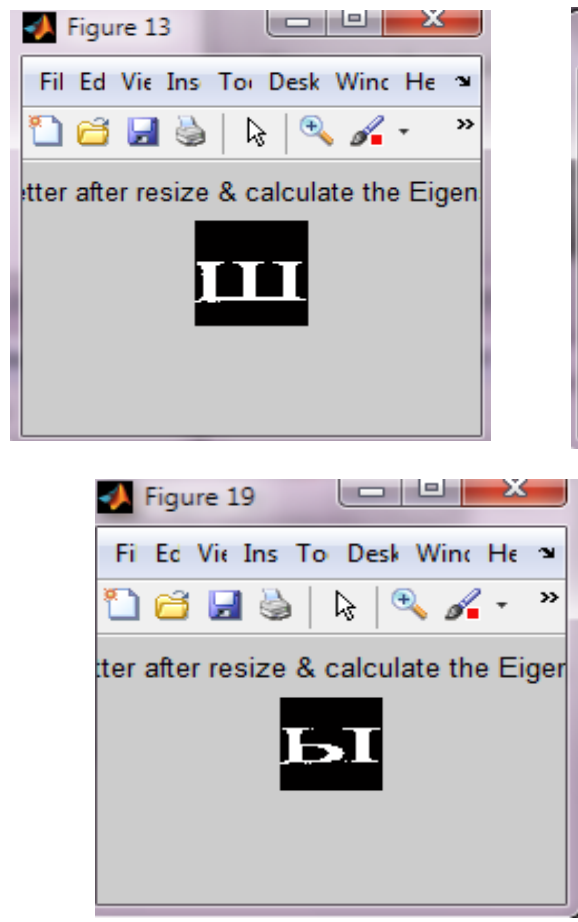

الثكل (10). شكل الحرف الروسي بعد عمليتي الدسح الأفقي والمسح العودي
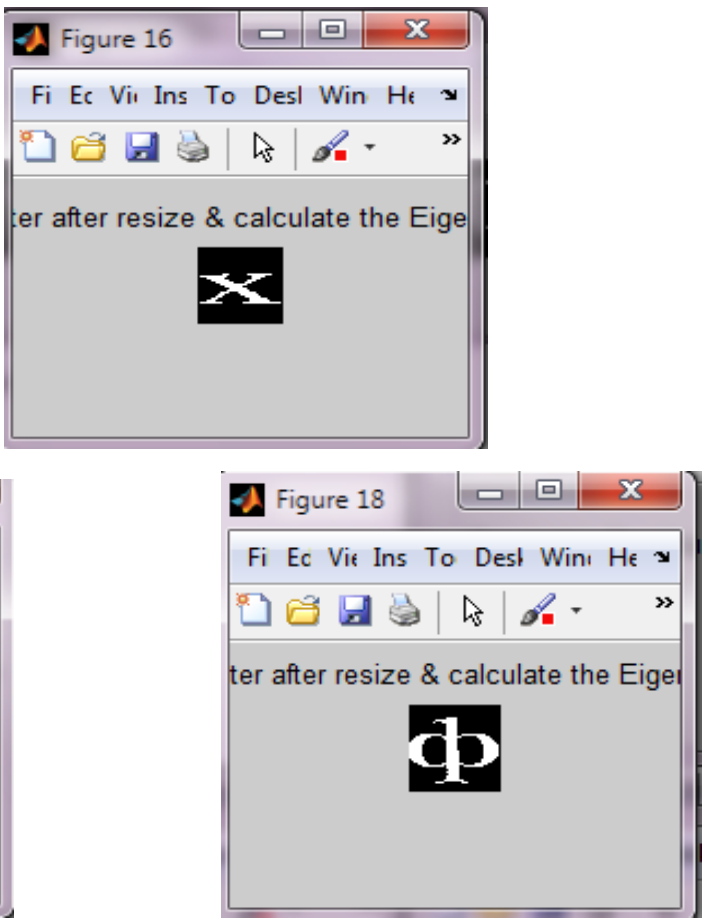

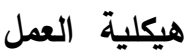

يعتمد نظام تمييز حروف اللغة الروسية على مجموعة البيانات المزودة لطبقة الإدخال والتي تم استخلاصها من صورة الحرف فيتم تدريب هذه الشبكة على البيانات ومن ثم اختبار الشبكة وعلى هذا الأساس فان الجانب العملي في البحث يبدأ بتحضير بيانات الإدخال ومعالجتها ثم اختبار الخوارزمية المستخدمة في التدريب لتحديد قيم الأوزان فتخزن ومن ثم يتم الاختبار حيث يقسم العمل إلى ثلاث مراحل منها:

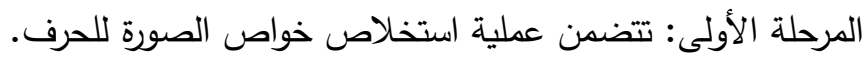
المرحلة الثانية: الاعتماد على الصفات المستخلصة وعدد المخرجات وتحديد معمارية الثبكة العصبية أيلمان .Elman

المرحلة الثالثة: في هذه المرحلة يتم تكوين ملف نصي يحتوي على النصوص التي تم تميزها والثكل (11) يمثل المخطط الصندوقي للجزء العملي. 


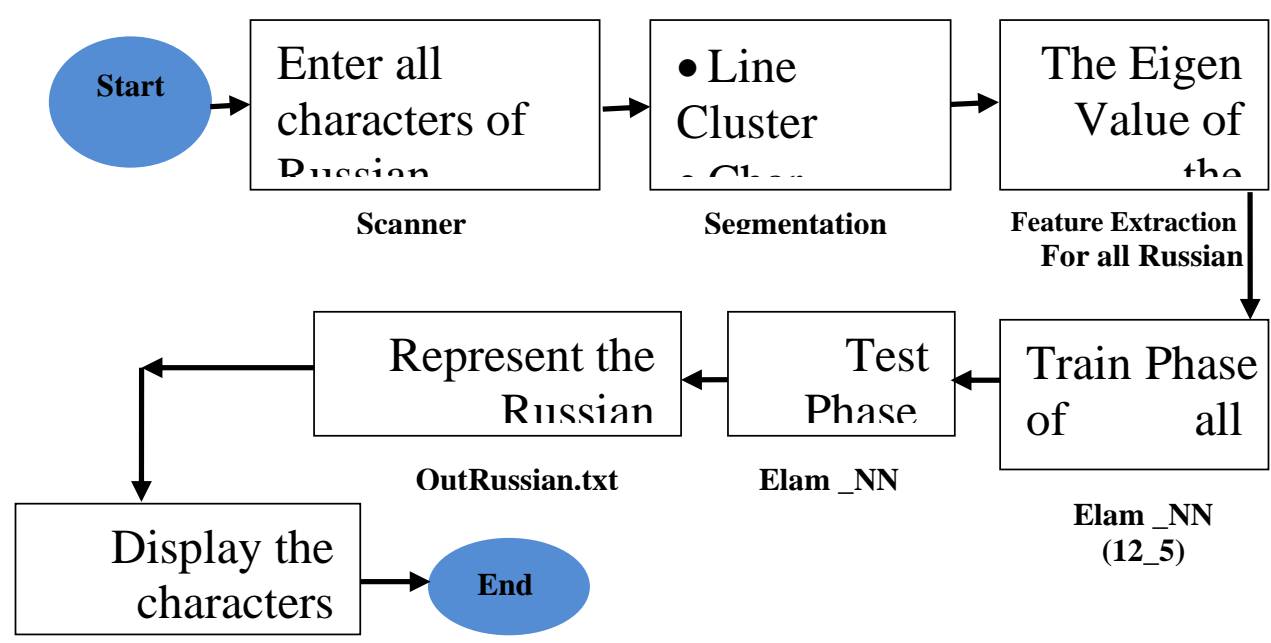

الثكل (11). المخطط الصندوقي للجزء العملي

5-7 - 5 5 إعداد إدخال البيانات

أن البيانات المدخلة على النظام تمثل القيم الذاتية (Eigen Value) لصورة الحرف، والتي تم الحصول

عليها من خلال دراسة وتحليل الصورة بعد تقسميها كما تم ذكره في المسح الأفقي والمسح العمودي وهي أثنا عشر قيم ذاتية (Eigen Value) لكل حرف حيث يتم استخدامها لتدريب الثبكة والتي سيتم توضيحها في الفقرة التالية.

$$
\text { الحروف الروسية وترشيح البيانات }
$$

أن نظام التمييز الصوري للحروف الروسية يستطيع ترشيح البيانات الداخلة على الثبكة إذا كانت من

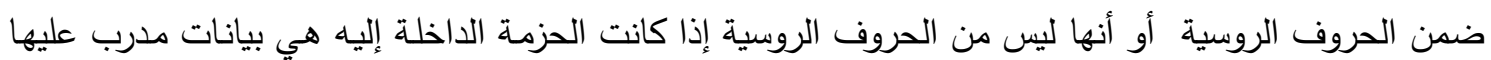
أو بيانات غير مدرب عليها أي غير معرف بالنسبة للنظام ويكون الإخراج (Target) بالنظام الثنائي بحيث يقابل كل حرف روسي رقم بالنظام الثنائي وكما موضح في معرب الجدول (2). الجدول (2). تمثيل الحروف بالنظام الثنائي

\begin{tabular}{|c|c|c|c|c|c|c|c|}
\hline Russian letter & b6 & b5 & b4 & b3 & bF2 & b1 & letter number \\
\hline$\ddot{\mathbf{E}}$ & 0 & 0 & 0 & 0 & 0 & 1 & 1 \\
\hline Й & 0 & 0 & 0 & 0 & 1 & 0 & 2 \\
\hline Ц & $\mathbf{0}$ & 0 & $\mathbf{0}$ & 0 & 1 & 1 & 3 \\
\hline $\mathbf{y}$ & $\mathbf{0}$ & 0 & $\mathbf{0}$ & 1 & 0 & 0 & 4 \\
\hline К & $\mathbf{0}$ & 0 & $\mathbf{0}$ & 1 & 0 & 1 & 5 \\
\hline $\mathbf{E}$ & $\mathbf{0}$ & 0 & $\mathbf{0}$ & 1 & 1 & 0 & 6 \\
\hline H & 0 & 0 & $\mathbf{0}$ & 1 & 1 & 1 & 7 \\
\hline$\Gamma$ & $\mathbf{0}$ & 0 & 1 & 0 & 0 & 0 & 8 \\
\hline ШII & $\mathbf{0}$ & 0 & 1 & 0 & 0 & 1 & 9 \\
\hline Щ & $\mathbf{0}$ & 0 & 1 & 0 & 1 & 0 & 10 \\
\hline 3 & $\mathbf{0}$ & $\mathbf{0}$ & 1 & $\mathbf{0}$ & 1 & 1 & 11 \\
\hline$X$ & $\mathbf{0}$ & $\mathbf{0}$ & 1 & 1 & 0 & 0 & 12 \\
\hline $\mathbf{b}$ & 0 & 0 & 1 & 1 & 0 & 1 & 13 \\
\hline$\Phi$ & $\mathbf{0}$ & 0 & 1 & 1 & 1 & 0 & 14 \\
\hline Ы & $\mathbf{0}$ & 0 & 1 & 1 & 1 & 1 & 15 \\
\hline B & $\mathbf{0}$ & 1 & $\mathbf{0}$ & $\mathbf{0}$ & 0 & 0 & 16 \\
\hline $\mathbf{A}$ & $\mathbf{0}$ & 1 & $\mathbf{0}$ & $\mathbf{0}$ & 0 & 1 & 17 \\
\hline П & $\mathbf{0}$ & 1 & $\mathbf{0}$ & $\mathbf{0}$ & 1 & 0 & 18 \\
\hline $\mathbf{P}$ & $\mathbf{0}$ & 1 & $\mathbf{0}$ & $\mathbf{0}$ & 1 & 1 & 19 \\
\hline
\end{tabular}




$\begin{array}{ccccccccc}\text { О } & \mathbf{0} & \mathbf{1} & \mathbf{0} & \mathbf{1} & \mathbf{0} & \mathbf{0} & \mathbf{2 0} \\ \text { Л } & \mathbf{0} & \mathbf{1} & \mathbf{0} & \mathbf{1} & \mathbf{0} & \mathbf{1} & \mathbf{2 1} \\ \text { Д } & \mathbf{0} & \mathbf{1} & \mathbf{0} & \mathbf{1} & \mathbf{1} & \mathbf{0} & \mathbf{2 2} \\ \text { Ж } & \mathbf{0} & \mathbf{1} & \mathbf{0} & \mathbf{1} & \mathbf{1} & \mathbf{1} & \mathbf{2 3} \\ \text { Э } & \mathbf{0} & \mathbf{1} & \mathbf{1} & \mathbf{0} & \mathbf{0} & \mathbf{0} & \mathbf{2 4} \\ \text { Я } & \mathbf{0} & \mathbf{1} & \mathbf{1} & \mathbf{0} & \mathbf{0} & \mathbf{1} & \mathbf{2 5} \\ \text { Ч } & \mathbf{0} & \mathbf{1} & \mathbf{1} & \mathbf{0} & \mathbf{1} & \mathbf{0} & \mathbf{2 6} \\ \text { С } & \mathbf{0} & \mathbf{1} & \mathbf{1} & \mathbf{0} & \mathbf{1} & \mathbf{1} & \mathbf{2 7} \\ \text { М } & \mathbf{0} & \mathbf{1} & \mathbf{1} & \mathbf{1} & \mathbf{0} & \mathbf{0} & \mathbf{2 8} \\ \text { И } & \mathbf{0} & \mathbf{1} & \mathbf{1} & \mathbf{1} & \mathbf{0} & \mathbf{1} & \mathbf{2 9} \\ \text { T } & \mathbf{0} & \mathbf{1} & \mathbf{1} & \mathbf{1} & \mathbf{1} & \mathbf{0} & \mathbf{3 0} \\ \text { b } & \mathbf{0} & \mathbf{1} & \mathbf{1} & \mathbf{1} & \mathbf{1} & \mathbf{1} & \mathbf{3 1} \\ \text { Б } & \mathbf{1} & \mathbf{0} & \mathbf{0} & \mathbf{0} & \mathbf{0} & \mathbf{0} & \mathbf{3 2} \\ \text { Ю } & \mathbf{1} & \mathbf{0} & \mathbf{0} & \mathbf{0} & \mathbf{0} & \mathbf{1} & \mathbf{3 3}\end{array}$

7-7 هيكلية الثبكة العصبية المستخدمة في النظام

أن هيكلية الشبكة يجب أن تتلاءم مع هيئة المسالة المراد حلها وفي هذا العمل فتحتاج الثبكة إلى أثنا عشر عقدة التي تمثل متجه الإدخال والتي تمثل الصفات الناتجة من القيم الذاتية (Eigen Value) وستة عقد هينه تمثل متجه الإخراج التي تكون كافية لتمثيل حالات الحروف المفحوصة حيث يتم أعداد وتهيئة الإدخال بما يلائم

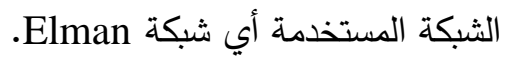

8-7 التمييز

بعد أن تم تدريب الثبكة أصبحت جاهزة للعمل كأداة في نظام التمييز الضوئي للحروف الروسية يكون عملها مشابه لمرحلة التدريب ويدعى هنا بمرحلة الفحص (الاسترجاع) وكذلك هيكلية الثبكة المستخدمة في مرحلة

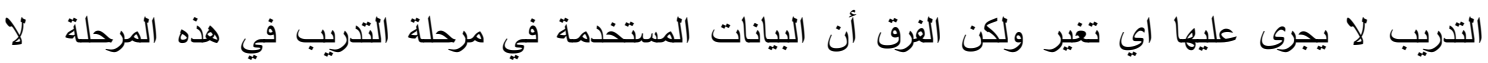
تستعمل في هذه المرحلة بدلا من ذلك تستعمل الأوزان المخزونة بعد استقرار الثبكة والحصول على الأوزان المثالية. وتعتبر بأنها أوزان ابتدائية وثابتة في الثبكة في هذه دالحالة تستطيع الشبكة تحليل الإدخال لكي تميز

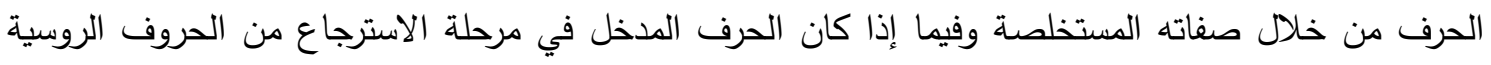
بنفس الخطوات التي أجريت في مرحلة التدريب. 9-7 تنفيذ النظام أن تتفيذ هذا النظام يكون أولا باستخلاص الصفات من صورة الحرف بعد مرحلتي المسح الأفقي والمسح

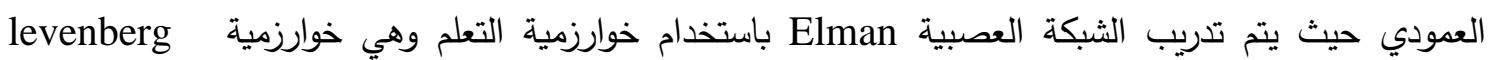

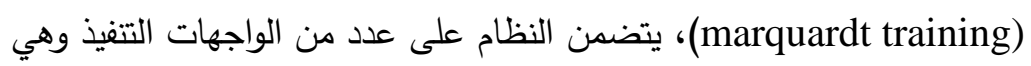

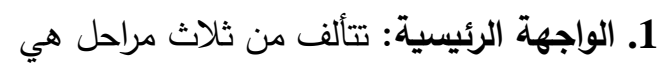
• المرحلة الأولى: الدخول إلى البرنامج حيث تفتح وتعمل على انتقال إلى واجهة تتفيذ البرنامج وكما موضح

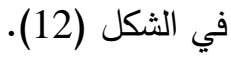




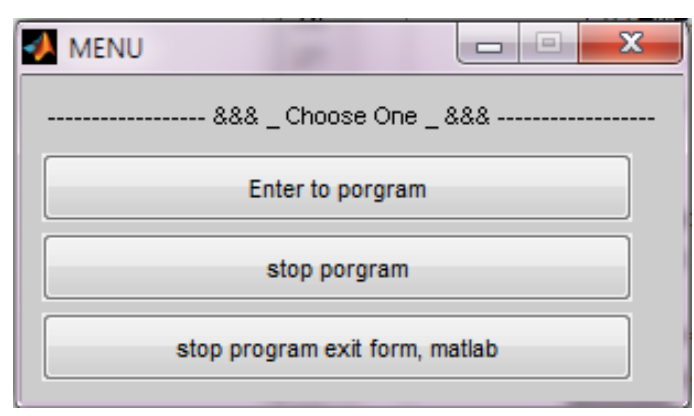

الثكل (12). واجهة تتفيذ البرنامج الرئيسية

• المرحلة الثانية: الخروج من البرنامج مع البقاء ضمن لغة ماتلاب (Matalb).

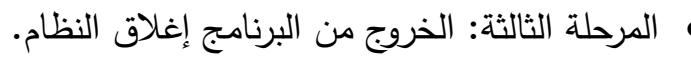
2. واجهة تنفيذ برنامج التمييز

أن الواجهة الثانية أيضاً تتكون من ثلاث مراحل الأولى فحص الصورة وتدريب الثبكة العصبية والاختيار الثاني يمثل اختيار الثبكة والاختيار الثالث يمثل العودة إلى الواجهة الرئيسية وكما مبين لانين

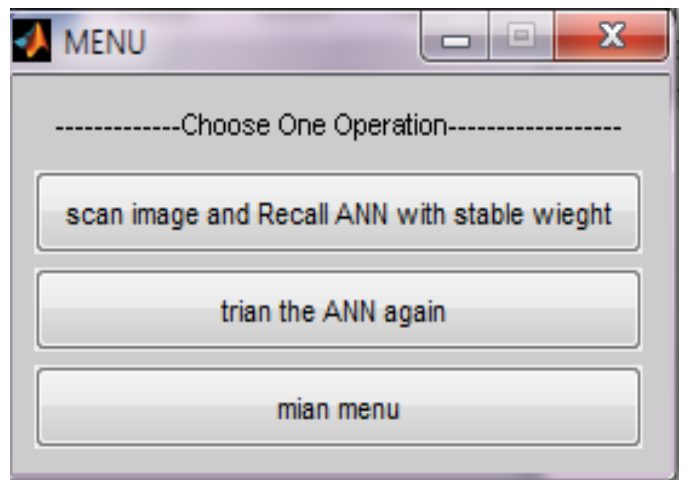

$$
\text { الثكل (13). واجهة البرنامج الثانوية }
$$

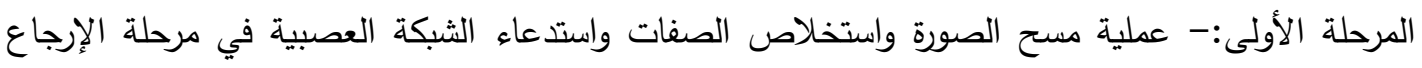
حيث يستدعى صورة الوثيقة من صيغة (Bmp.) وكما مبين في الثكل التالي حيث يتم تقسيم الأسطر إلى حروف وإيجاد القيم الذاتية لكل حرف ومن ثم استرجاع للشبكة العصبية المدربة مع الأوزان المستقرة وتصنيفها لإخراج الثبكة لكي تميز الثبكة العصبية الحروف الروسية ويتم خزنها في ملف نصي.

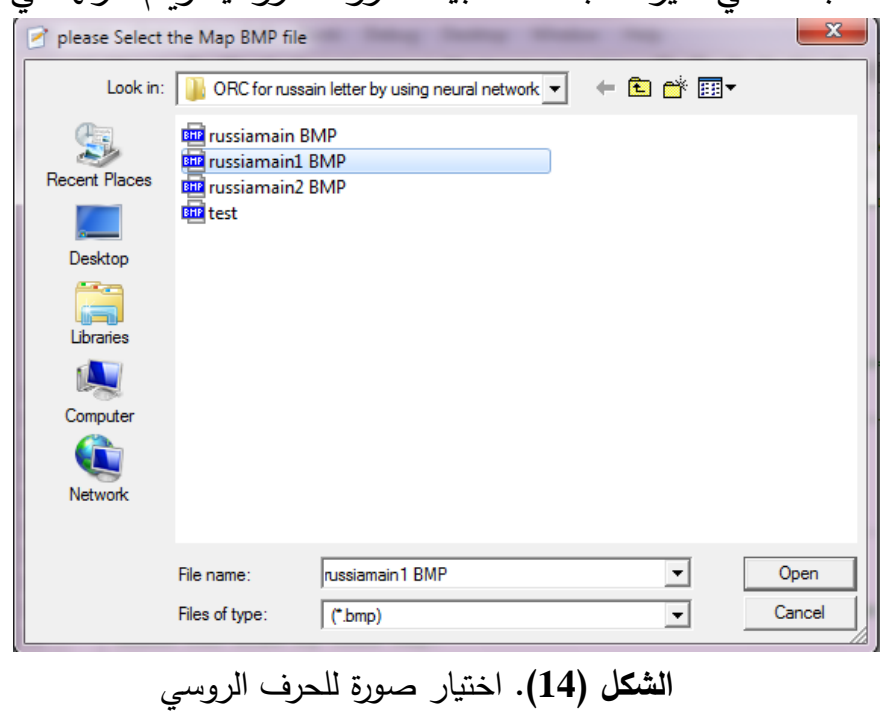

الثكل (14). اختيار صورة للحرف الروسي 
المرحلة الثانية:- تدريب الثبكة العصبية مرة ثانية وتم اعتماد نسبة الخطأ 0.097 وعدد الدورات 1000، وذلك لغرض لو أن الثبكة العصبية يتم فيها تدريب النوع الجديد من الرموز مع ملاحظة التطوير المستقبلي. المرحلة الثالثة:- العودة إلى الواجهة الرئيسية للبرنامج.

أن تنفيذ الواجهات المذكورة في كل من الأثكال (12) ،(13) و(14) كانت النتائج كالآتي

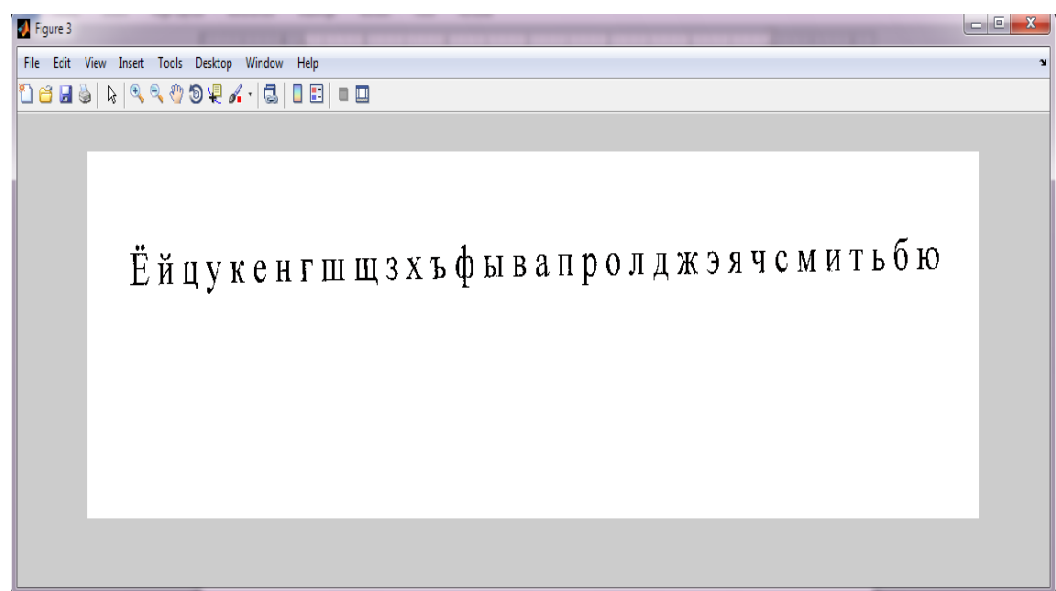

الثكل (15). اختبار الحرف الروسي

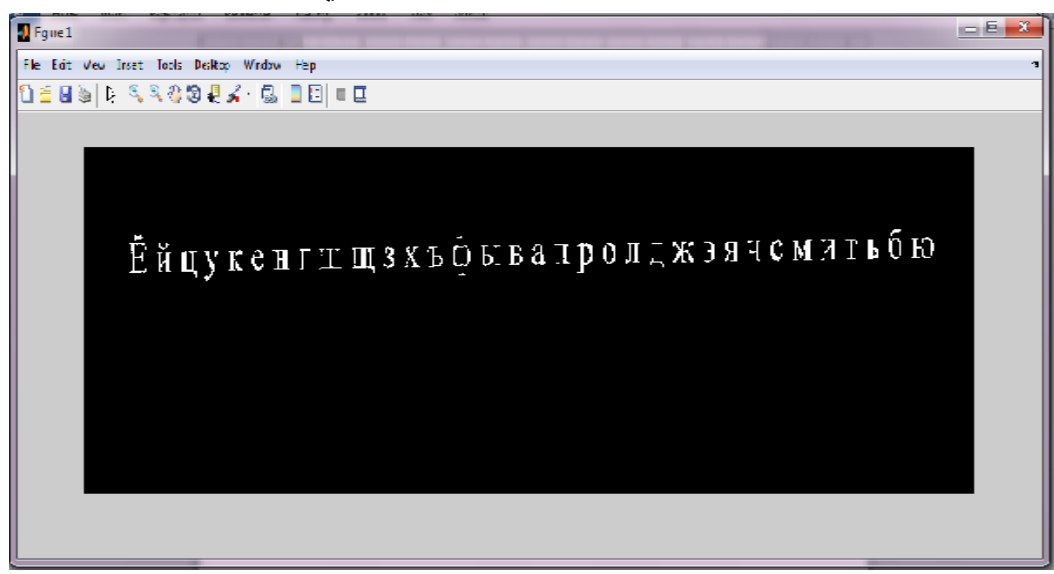

الثكل (16). صورة الحرف

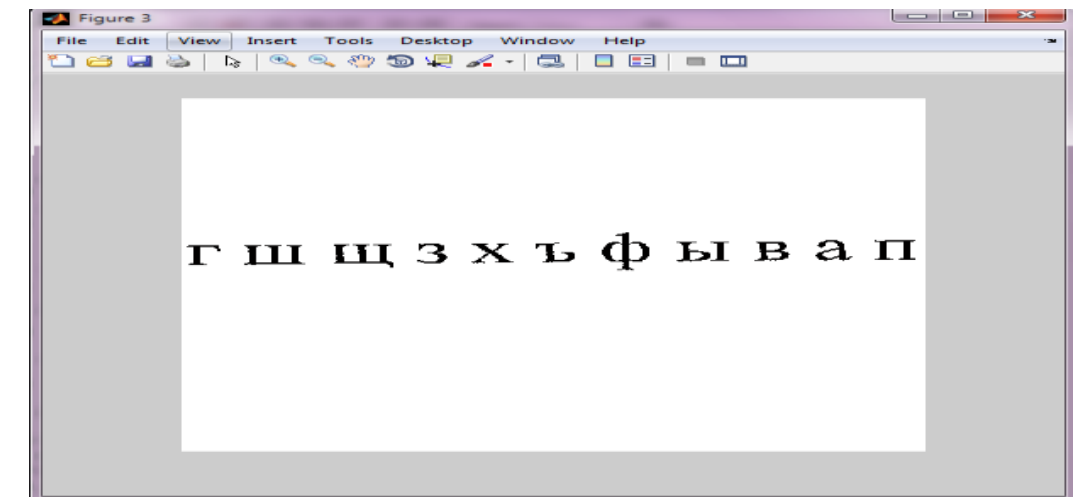

الثكل (17). OutRussian.txt ملف صوري لجزء من الأحرف الروسية في ملف نصي 
وبعـــت تنفيـذ النظـام بالكامـل علـى الصــورة السـابقة في الثـكل (12) وخـزن النتـائج فـي ملـف خـارجي

First space in image $=1$

OutRussian.txt

Virtcal size of font $=600$

Space between line $=0$

Number of line in Image $=1$

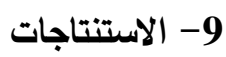

أن النظام الحاسوبي الذي تم بناؤه باللغة البرمجية ماتلاب (Matlab) ويقوم بقراءة الملف الصوري لحرف

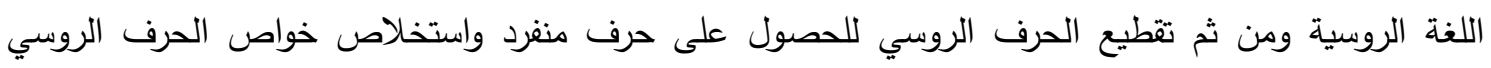
بالاعتماد على القيم المجزة للحرف(Eigen Value) والتي تنتج قيم حقيقية وقيم خيالية فيتم معالجة القيم الخيالية وذلك باستدعاء الدالة (image) في لغة ماتلاب (Matlab) أو قسمة العدد الخيالي على الجذر التربيعي للعدد(-1) ) وهو الجذر الخيالي is

أن بناء شبكة أيلمان لتمييز الحرف الروسي في مرحلتي التدريب والفحص وبذلك نحصل على ملف نصي

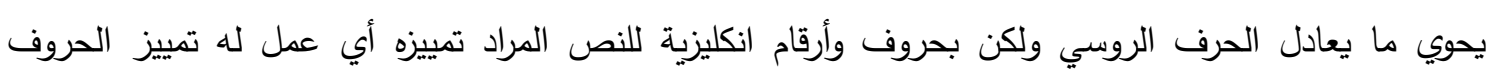

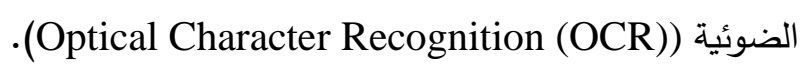

أن النتائج التي تم الحصول عليها من خلال تتفيذ النظام كانت كفوة وكان التمييز باستخدام الثبكة

العصبية أيلمان Elman حقق نجاح بنسبة تمييز وصلت إلى 90 \% لهيئتي الحرف الروسي (الكبيرة والصغيرة)

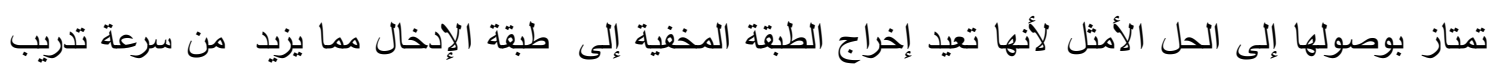

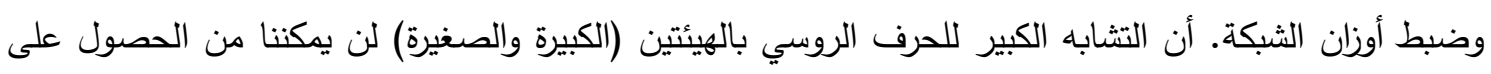
نسبة التمييز البالغة 95 \% والتي حققها كل من الباحثون (جمال صلاح، طارق طه) في تمييز الحروف العبرية باستخدام الثبكات العصبية إيلمان وذلك لعدم احتمالية أوجه التشابه بالنسبة الحروف العبرية. أما الباحثون (جمال صلاح الدين، أسيل وليد) في بحث تمييز الحروف اللاتينية باستخدام الثبكات العصبية (شبكة الانتشار العكسي) حصلوا على نسبة تمييز 82.75 \%. 
المصادر

[1] Urada, J.M., "Introduction to artificial neural systems", JAICO publishing House, Mumbai, (1994).

[2] Kosko B., "Artificial neural network and fuzzy system", A Hall, inc. Englewood Gliffs, N.J., (1991).

[3] J. can - Louis , L. " Problem solving and artificial intelligence", BPCC wheaston Ltd., Exeter, London .(1990).

[4] Majeed.Jamal Salahadeen,AlNeamy, "Pattern Recognition Approach for Arabic Letter Processing " M.Sc. computer Science,Computer and Mathematic Science, University of Mosul, Iraq.(1997).

[5] JohnVince, " Mathematics for Computer Graphics", MTech, PhD, DSc, FBCS, CEng, Media School, University of Bournemouth, Talbot Campus, Fern Barrow, pool Bh12 5BB,Uk, Library of Congress control Number, (2005).

[6] Sanjeev Kunte .R and Sudhaker Samuel.R.D., "Asimple and efficient optical character recognition system for basic symbols in printed Kannada text", Department of Electronics and Communication, S J college of engineering, (2006). http://www.academic.research

[7] Christopher M.B, “ Neural networks for Pattern Recognition”, Department of Computer Science and Applied Mathematics, Aston Universty, (1995).

http://www.academic.research

[8] Beezer, R A., "A first course in linear algebra", free online book under GNU license, University of Puget sound ,(2006)

http://www.linear.ups.edu

[9] Roman steven, “Advanced linear algebra (3 ${ }^{\text {rd }}$ ed.)", Newyork, Ny:Springer science+Business media, LLC, ISBN 978-0-387-72828-8, (2008).

[10] Matlab/R2007b/Help/Historical Networks (Neural Network Toolbox).

[11] eignvalues and eigenvectors

http://www.en.wikipedia.org/wiki/eignvalues and eigenvectors.

خليل، بيداء إبراهيم، ’'مقارنـة بين الشبكات العصبية 6،، رسالة ماجستير، جامعة الموصل، كلية علوم الحاسبات والرياضيات، قسم علوم الحاسبات، العراق، (2002).

م. عـلام زكي عيسى، 'الثـبكات العصبية - البنيـة الهندسية - الخوارزميـات -التطبيقـات،، دار

الثعاع للنشر ، سوريا - حلب - الطبعة الأولى، (2002).

السبتي، د.جوزيف ضايف، "الجبر الخطي،، جامعة البصرة، دار الحكمة، (1988)، ص 212.

سيد مجيد، جمال صـلاح الدين، طـه، طـارق حازم ومحمد، أسامة ياسين، ’تمييز الحروف العبريـة

باستخدام الثبكات العصبية ، ، جامعة الموصل، كلية علوم الحاسبات والرياضيات، كلية الحدباء

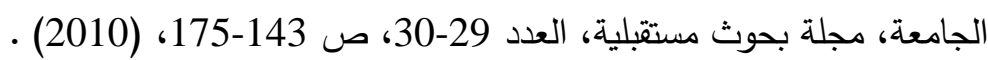

سيد مجيد، جمال صـلاح الدين، علي، أسيل وليد وسيد مجيد، عمار صـلاح الدين، 'تمييز الحروف

اللاتينيـة باسـتخدام الثـبكات العصبية،،، جامعة الموصل، كليـة علوم الحاسبات والرياضـيات، مجلـة

الرافدين لعلوم الحاسبات والرياضيات، المجلد 4، العدد 2، (2007). 


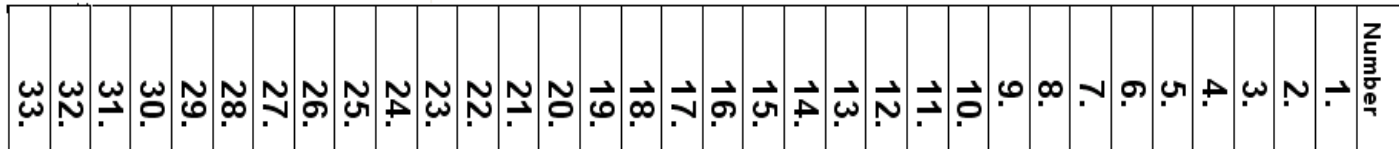

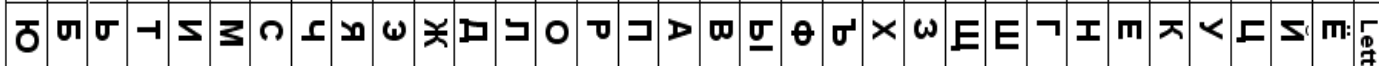

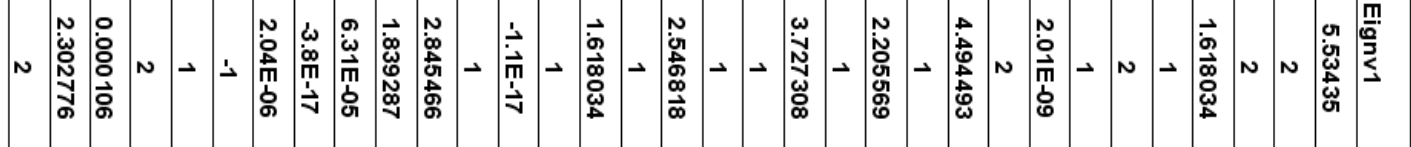

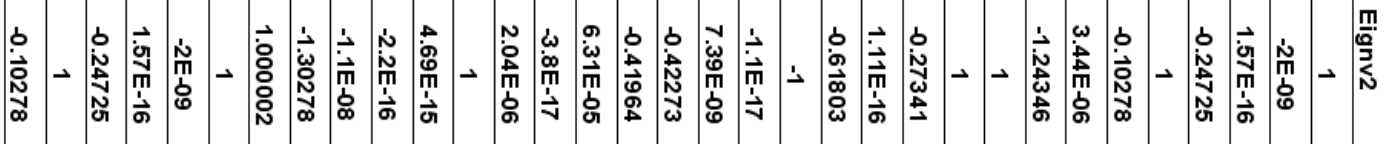

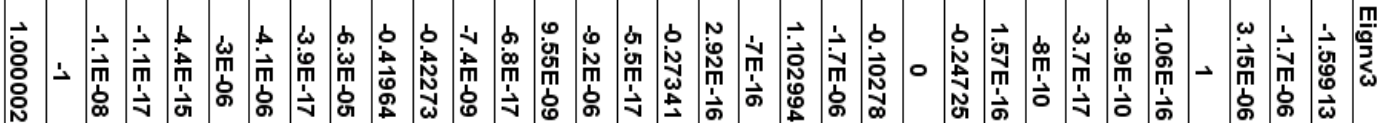

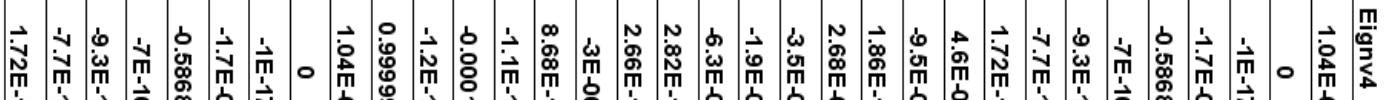

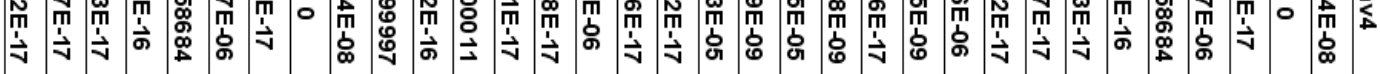

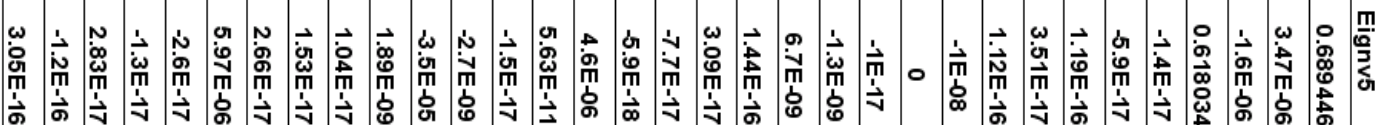

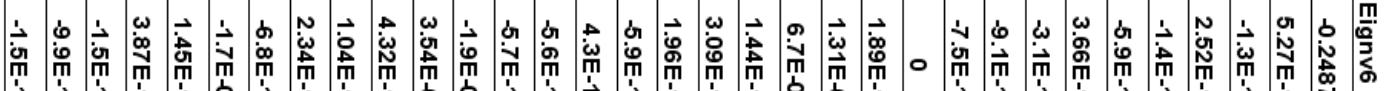

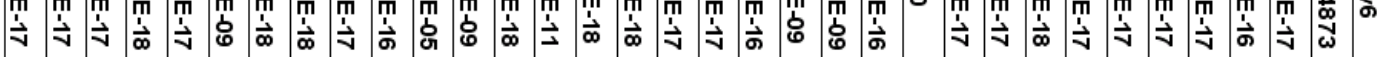

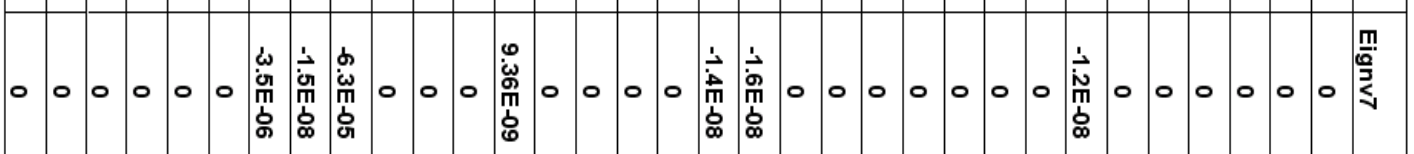

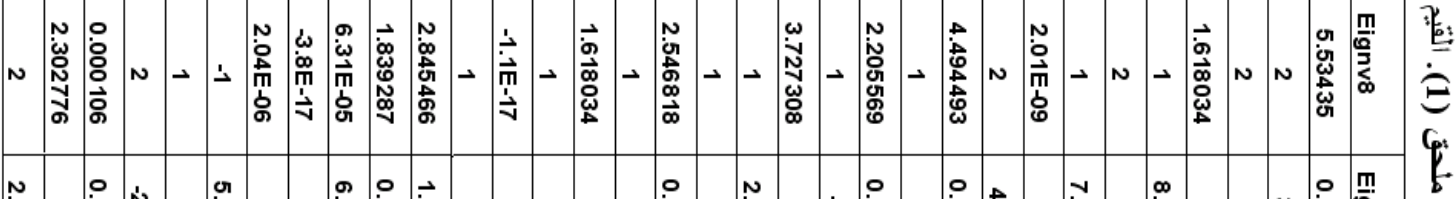

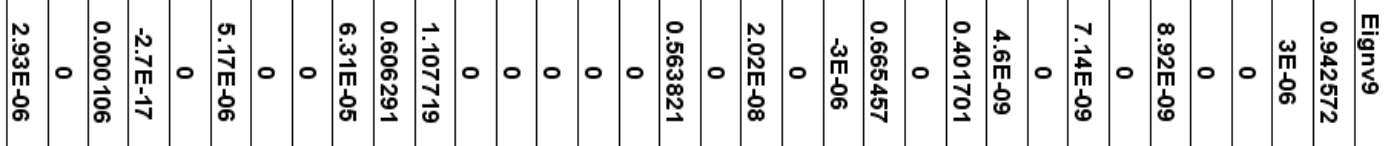

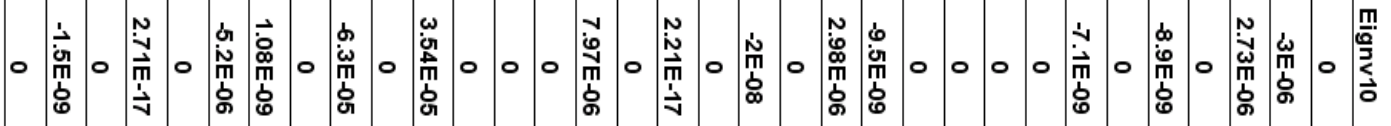

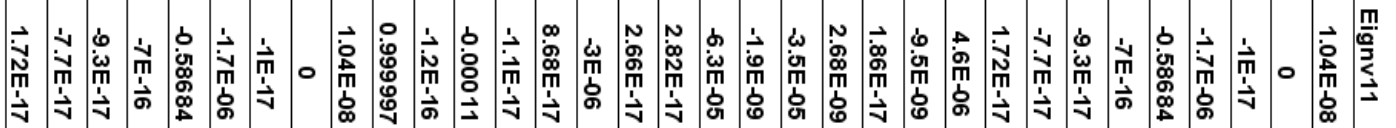

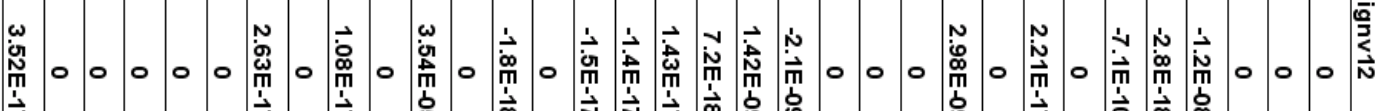

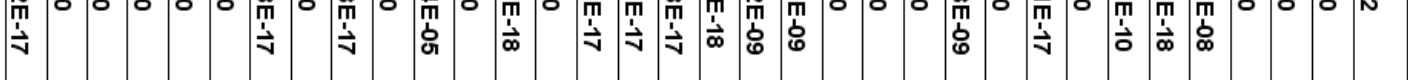

